\title{
Auditory Brainstem Implants
}

\section{S Raghunandhan, Mohan Kameswaran}

\author{
Consultant ENT Surgeon, Madras ENT Research Foundation, Chennai, Tamil Nadu, India
}

Correspondence: S Raghunandhan, Consultant ENT Surgeon, Madras ENT Research Foundation, No. 1, 1st Cross Street Off. 2nd Main Road, Raja Annamalaipuram, Chennai-600028, Tamil Nadu, India, Phone: (044)24311411/412/413/414/415 e-mail: merfmk30@yahoo.com

\begin{abstract}
Auditory brainstem implants (ABIs) are currently indicated for patients with neurofibromatosis type 2 (NF-2) tumors involving both vestibulocochlear nerves. The ABI helps bypass the damaged cochlear nerves and stimulates the cochlear nucleus in the brainstem directly thereby restoring auditory sensation. The implant is usually placed in the lateral recess of the fourth ventricle after tumor resection. The indications for $\mathrm{ABI}$ have recently expanded onto even nontumoral cases, such as congenital bilateral cochlear nerve aplasia. In such cases, the $\mathrm{ABI}$ helps bypass the nonfunctioning hypoplastic or absent cochlear nerves and stimulates the cochlear nucleus directly thereby restoring auditory sensation. This article reviews the nuances of this sophisticated implant, shares our experience with auditory brainstem implantation and its current status in world literature.
\end{abstract}

Keywords: Auditory brainstem implant (ABI), Neurofibromatosis 2 (NF-2), cochlear nerve aplasia.

\section{INTRODUCTION}

Auditory brainstem implants are safe and effective in rehabilitation of hearing in certain retrocochlear disorders as in neurofibromatosis type 2 or congenital cochlear nerve hypoplasia/aplasia, wherein a cochlear implant would be ineffective. Multidisciplinary collaboration between neurologist, neurosurgeon, implant audiologist and neuroanesthetist is required in order to perform this intricate and sophisticated surgery.

Researchers at the House Ear Institute (HEI), in USA where the ABI was initially developed, agree that most patients with the implant have good auditory awareness with appreciation of environmental sounds, but obtain more modest benefit with regard to speech perception. Majority of ABI patients use the implant, in order to facilitate lip reading while some can, in varying degrees, comprehend speech directly. It has been demonstrated that the ABI with surface electrodes may provide sufficient stimulation of the central auditory system in adults for open-set speech recognition. These favorable results motivated the clinicians to extend $\mathrm{ABI}$ indications onto children with profound hearing loss who were not a candidate for a CI. The indications for ABI have expanded by leaps and bounds over the last ten years onto children and adults with nontumoral (NT) cochlear nerve abnormalities, which cannot be benefited from cochlear implantation.
It is notable that the incidence of cochlear nerve aplasia in the overall population world over is very low, estimated at one in every 100,000 newly born babies wherein ABI is indicated. In 2000, the FDA approved use of auditory brainstem implants (ABIs) in adults and children over the age of 12 years, with bilateral NF-2 tumors (tumoral cases). Here, the vestibulocochlear nerve needs to be sacrificed during tumor excision and the ABI may be placed within the brainstem at the same sitting or later. The array of ABI electrodes, directly stimulate the auditory fibers of the cochlear nucleus within the lower brainstem, bypassing the peripheral auditory system. In the present day, about 500 people have received the ABI around the world and the benefit has proved to be substantial (Figs 1A and B).

\section{CLINICAL WORK UP}

A meticulous work up is mandatory, prior to selection of ideal cases for ABI. In children, a detailed genetic study, neurological and psychomotor assessment is necessary apart from the routine work up as done for cochlear implantation. High resolution radioimaging with CT/MRI of the brain and inner ear often provides valuable clues to the operating team, for planning the side of implantation and the surgical approach. Advanced objective electrophysiological hearing tests need to be performed in order to assess the functions of the brainstem and higher auditory centers. Extensive 

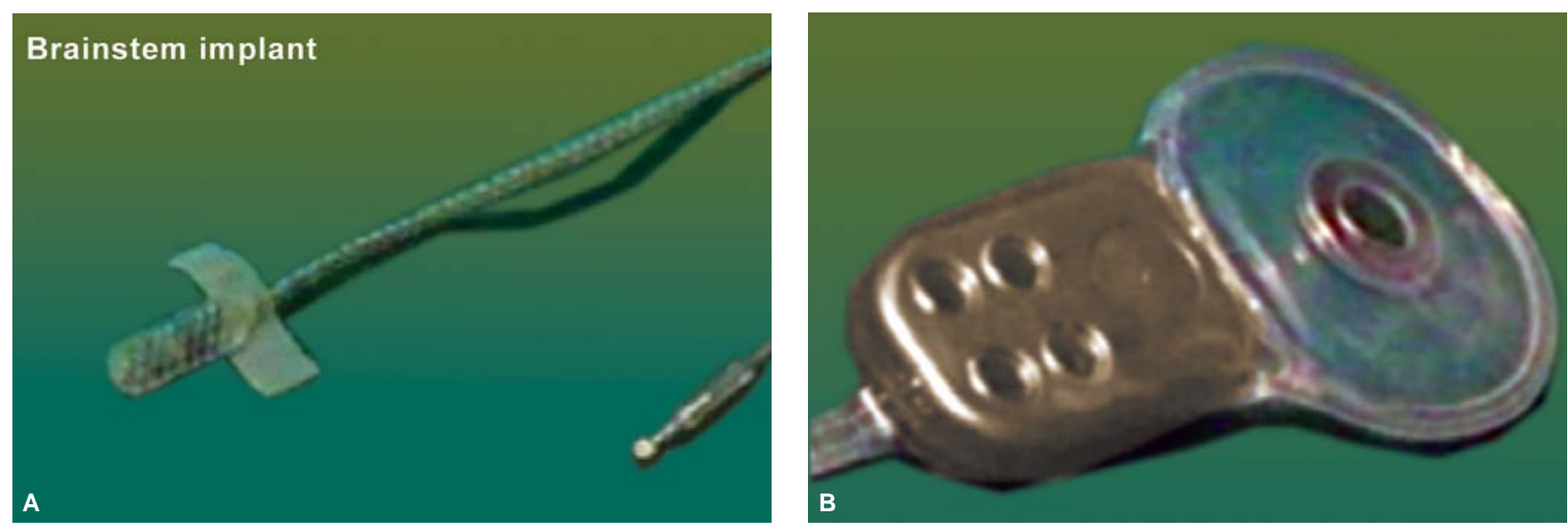

Figs $1 \mathrm{~A}$ and $\mathrm{B}$ : Two ends of the auditory brainstem implant

counseling for the parents and family, regarding the details of surgery, habilitation process and the expected outcomes of the ABI is vital.

\section{THE OPERATIVE PROCEDURE}

Under intensive neuroanesthesia with intraoperative cranial nerve monitoring, the patient is positioned head and neck flexed in order to gain good exposure of the occipital and retromastoid regions. A reverse hockey stick incision is made in the retromastoid region, in order to expose the squamous part of occipital bone and temporal bone. A burrow is made at the asterion using the craniotome. Craniotomy exposes the transverse sinus superiorly and sigmoid sinus laterally. Dura needs to be opened by a vertical incision $1 \mathrm{~cm}$ away from the sigmoid sinus and is reflected laterally. CSF is let out from the basal cisterns to make the cerebellum lax. Cerebellum is retracted medially to reach the CP angle where the VII and VIII nerve complex is identified. Inferiorly the lower cranial nerves are seen and followed medially onto the foramen of Luschka where the choroids plexus is identified. Further dissection is done to reach the floor of the IV ventricle, where a constant vein called the straight vein is present, which leads to the site of the cochlear nucleus (Fig. 2).

In tumoral cases as in NF-2, tumor excision via the same approach precedes the implantation. After tumor excision, the landmarks (seventh, eighth and ninth cranial nerves, choroid plexus) for the foramen of Luschka are identified. Location of the lateral recess can be confirmed by noting the egress of CSF during Valsalva maneuver. The $\mathrm{ABI}$ electrode array is then inserted into the lateral recess and positioned once the cochlear nucleus is well-delineated



Fig. 2: CP angle showing facial nerve with hypoplastic vestibulocochlear nerve bundle

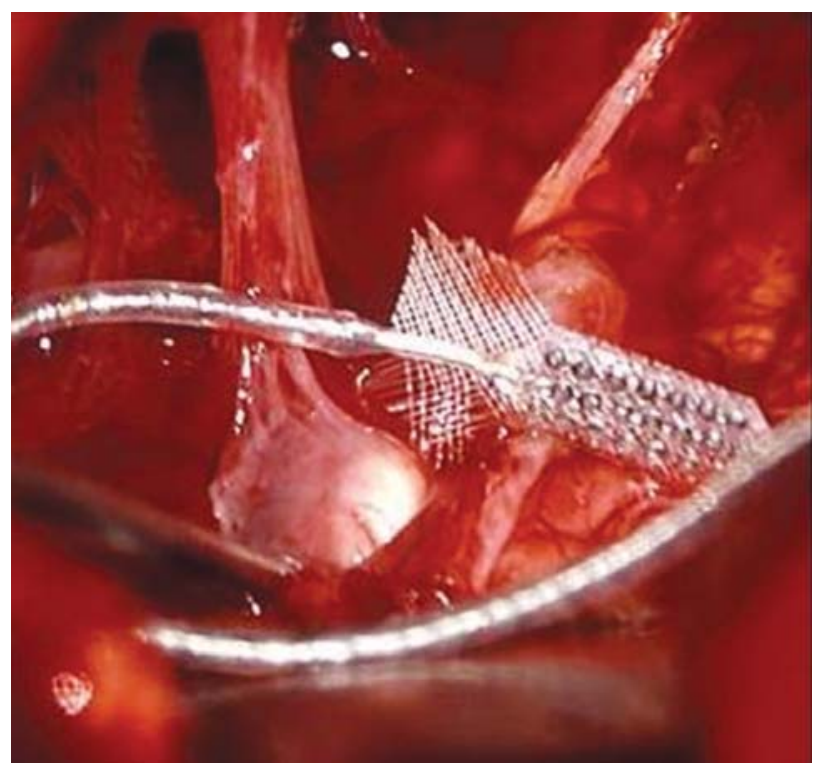

Fig. 3: Insertion of electrodes in brainstem 
(Fig. 3). Initially temporary electrodes are placed on it and electrically evoked auditory brainstem responses (EABRs), early midlatency responses (EMLRs) and device telemetry (DT) are performed to check the optimal positioning and functioning of the electrodes. Once integrity is confirmed, the permanent electrodes are then placed onto the cochlear nucleus and positioned with fibrin glue and surgical.

The receiver-stimulator coil is placed in a bed created in the area postero-superior to the craniotomy. It is placed at least $10 \mathrm{~mm}$ behind the edge of the auricle and above the canthomeatal line and is angled 30 to 45 degrees posterosuperiorly. Tie-down holes are made on either side of the receiver stimulator for securing the implant. Reconfirmation of implant function is done with electrophysiological tests. The stimulus to ABI is delivered by an external component comprising of a microphone, a signal processor and a transmitter coil very similar to the cochlear implant. Dura is closed primarily in a water-tight fashion. Postoperative neuro-ICU care is necessary with cranial nerve monitoring.

The device is switched on 2 months after implantation, providing sufficient time for wound healing and full recovery of the convalescing patient. Switch on needs to be done in the operation theater with neural monitoring and adequate preparation for active CPR, as there may be inadvertent stimulation of other brainstem nuclei and the possibility of nonauditory stimulation of vital centers. The stimulus threshold and comfort level on each electrode is ascertained. Postoperative CT scan and X-ray skull confirm the position of the ABI in situ (Fig. 4).

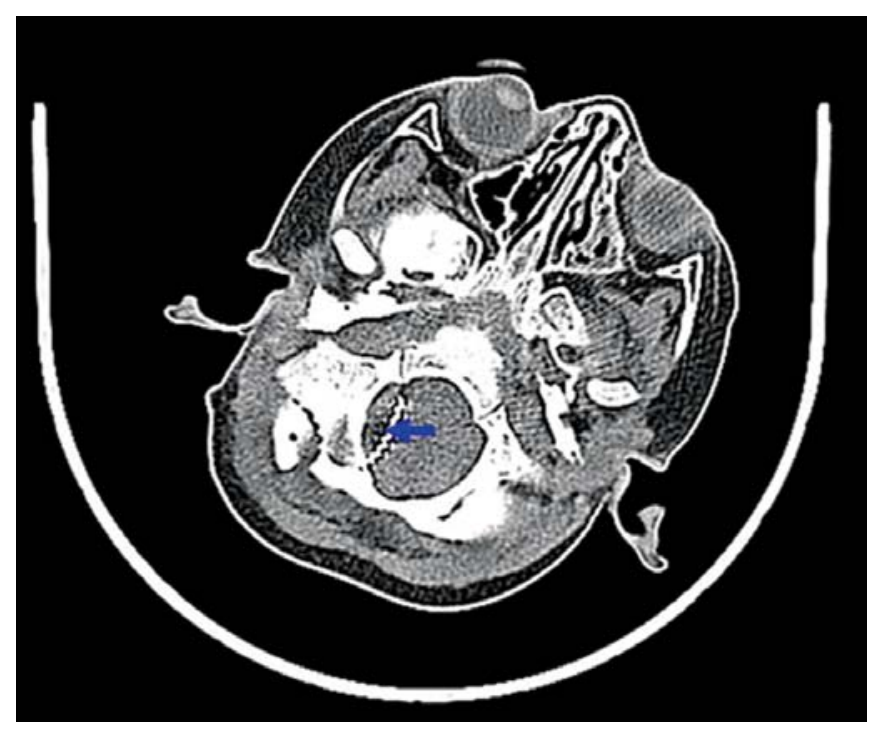

Fig. 4: Bainstem implant in situ
Intensive auditory verbal habilitation is then initiated and continues for a minimum period of one year (as advocated for CI) with periodical EABR and EMLR tests done during the follow-up for confirmation of device integrity and assessment of optimal performance the implantee. Most implantees develop very good sound awareness and good gross auditory discrimination with appropriate habilitation. Achievement of lucid environmental sound perception with pitch discrimination for closed set speech is often the culmination of the habilitation process.

\section{OUR EXPERIENCE}

Madras ENT Research Foundation, Chennai a premier Implant Institute in India was the first center in South Asia to perform ABI surgeries. The first AB Implantee was a 16 years old girl with bilateral NF-2 tumors. In 2005, she underwent tumor excision and implantation in the same sitting. She is presently doing remarkably well-pursuing a career in microbiology. The second implantee was a two years old child, born with bilateral cochlear nerve aplasia. In 2007, he underwent ABI, has completed successful habilitation program at our institute and is presently attending normal school. In our experience with habilitating these two AB Implantees, both patients have developed gratifying results, with good auditory awareness and speech recognition. The ABI is greatly aiding their lip-reading abilities and significant improvement is noted in their communication skills and quality of life.

\section{DISCUSSION}

Multichannel auditory brainstem implants (ABIs) are currently indicated for patients with neurofibromatosis type II (NF-2) and schwannomas involving the internal auditory canal or cerebellopontine angle. ${ }^{1,2}$ Increasingly, ABI is also being considered for nontumoral cases as in children with congenital cochlear nerve aplasia before the loss of neuronal plasticity. The current criteria for ABI include evidence of bilateral seventh and eighth cranial nerve tumors involving IAC or cerebellopontine angle, language competency, age $>12$ years or older, psychologic suitability, willingness to comply with research follow-up protocol and realistic expectations. Several factors can affect the benefit received from an ABI. These factors may include size and location of auditory nerve tumors, degree and duration of hearing loss prior to surgery, and commitment and motivation to use the $\mathrm{ABI} .^{3}$ 
Preoperative counseling regarding the importance of such factors as expectations, personal motivation, and family support is invaluable and the importance of regular use of the implant has to be stressed in order to maximize the benefits from the implant. For successful ABI surgery, a few important issues such as patient selection, choice of device, choice of approach, technique of tumor removal, knowledge of microanatomical variations, intraoperative identification of the cochlear nucleus and prevention of complications have to be considered.

There are several approaches described for tumor removal and placement of ABI. Translabyrinthine approach provides optimal access for both removal of the tumor and placement of the electrode array. A translabyrinthine approach is used by ENT surgeons and has the advantage of offering the best lateral angle to the exit of the cochlear nerve, allows the early identification of the facial nerve and does not require cerebellar retraction. Disadvantages with translabyrinthine approach are limited exposure of cranial nerves and vessels in the posterior fossa. The lateral suboccipital approach is preferred by neurosurgeons it is fast, safe and offers very good exposure of the lateral posterior fossa. The problems are cerebellar retraction and late identification of facial nerve. The angle to the lateral recess, the direct vision of the entrance is more difficult than in the translabyrinthine approach.

The middle cranial fossa approach is only possible with angled endoscopes; however, it is technically the most difficult and places the facial nerve at greatest risk. Penetrating electrodes have also been tried on the cochlear nucleus. This implant has the potential of offering improved performance due to better access to the complex tonotopic organization of the cochlear nucleus. Endoscopic guided placement of electrodes through retrosigmoid approach may also be of help in reducing the morbidity of surgery.

ABI appear to be more effective in children and adults with nontumor diseases of the auditory nerve or cochlea than in patients with NF-2 tumors. ${ }^{4}$ In 2006, Colletti reviewed the results of 80 patients fitted with ABIs at the University of Verona, Italy. One year after implantations, nontumor adults scored an average of 59 percent on openset speech perception tests, while tumor patients scored an average of only 11 percent. According to Dr. Colletti, a significant number of nontumor patients were able to understand speech at a level comparable to that of the most successful cochlear implants users including conversational telephone use. Nontumor patients had better hearing outcomes than tumor patients when the variation in the auditory benefit with the ABI in relation to the patient's underlying pathological conditions were taken into consideration. ${ }^{5,6}$

In the near future, it may be used in bilateral temporal bone fractures and demyelinating diseases affecting the eighth cranial nerves but sparing atleast one cochlea nucleus. Auditory brainstem implantation has also been recommended for use in cases of bilateral totally ossified cochlea in which a cochlear implant cannot be used. ${ }^{7}$ Contraindications to ABI include previous stereotactic radiotherapy which has the risk of radiation necrosis of the cochlear nucleus region and anatomic distortion and fibrosis. ABI may not be possible in very large tumors which cause distortion of the brainstem.

\section{CONCLUSION}

Auditory brain stem implants have been used in NF-2 patients with bilateral vestibular schwannomas. The primary aim of treatment of patients with NF-2 is the recovery of hearing after reducing or extirpating the tumor. New evidence suggests that the ABI is also very effective in nontumoral patients particularly in prelingually deaf children with significant cochlear abnormalities with preclude cochlear implantation. Ideal patient selection for ABI is important and appropriate preoperative counseling regarding realistic expectations is mandatory with special emphasis on the importance of regular usage by the implantee with intensive auditory verbal habilitation.

\section{REFERENCES}

1. Otto SR, Brackmann DE, Hitselberger W. Auditory brainstem implantation in 12- to 18-year-old. Arch Otolaryngol Head Neck Surg May 2004;130(5):656-59.

2. Kanowitz SJ, Shapiro WH, Golfinos JG, Cohen NL, Roland JT Jr. Auditory brainstem implantation in-patients with neurofibromatosis type 2. Laryngoscope. Dec 2004;114(12): 2135-46.

3. Sollmann WP, Laszig R, Marangos N. Surgical experiences in 58 cases using the Nucleus 22 multichannel auditory brainstem implant. The Journal of Laryngology and Otology. Dec 2000;114(27):23-26. 
4. Colletti V, Carner M, Fiorino F, Sacchetto L, Miorelli V, Orsi A, Cilurzo F, Pacini L. Hearing restoration with auditory brainstem implant in three children with cochlear nerve aplasia. Otol Neurotol Sep 2002; 23(5):682-93.

5. Colletti V, Fiorino FG, Carner M, Miorelli V, Guida M, Colletti L. Auditory brainstem implant as a salvage treatment after unsuccessful cochlear implantation. Otol Neurotol Jul 2004;25(4):485-96.
6. Colletti V. Auditory outcomes in tumor vs. non-tumor patients fitted with auditory brainstem implants. Advances in Otorhinolaryngology, 2006;64:167-85.

7. Grayeli AB, Bouccara D, Kalamarides M, AmbertDahan E, Coudert C, Cyna-Gorse F, Sollmann WP, Rey A, Sterkers O. Auditory brainstem implant in bilateral and completely ossified cochlee. Otol Neurotol. Jan 2003;24(1): 79-82. 